\title{
TRATAMENTO DE 75 PACIENTES PORTADORES DE MALÁRIA PELO P. FALCIPARUM, EM PORTO VELHO (RO), COM MEFLOQUINA.
}

\section{João Barberino Santos e Aluízio Prata}

\begin{abstract}
Foi avaliada a resposta terapêutica em 75 doentes com malária pelo P. falciparum, em Porto Velho-Rondônia, tratados com mefloquina nas doses de 1250, 1000 e $750 \mathrm{mg}$. Não houve diferença significante nos resultados obtidos nos três grupos. Até o terceiro dia de tratamento, a febre desapareceu em $76,0 \%$ dos casos. A parasitemia negativou-se em $68,0 \%$ dos pacientes nos 3 primeiros dias após o início do tratamento, em $24,0 \%$ no quarto dia e em $5,3 \%$ no quinto. Havia um doente positivo no sexto dia e outro no sétimo. Nenhum paciente apresentou trofozoito no período de seguimento de 7 a 28 dias. Não houve modificaçōes do quadro hematológico, exceto por um incremento da taxa de reticulócitos em $76,0 \%$. As alterações bioquímicas registradas no seguimento dos doentes constaram de elevação da desidrogenase lática $(28,0 \%)$ e, da fosfatase alcalina (41,3\%). As reações colaterais foram discretas e infreqüentes; em $72,0 \%$ dos tratados não se constatou nenhum efeito secundário. Não se verificou nenhum caso de recidiva. A mefloquina revelou-se eficaz e bem tolerada no tratamento da malária no Estado de Rondônia.
\end{abstract}

Palavras-chaves: Malária. Mefloquina. Tratamento.

A resistência medicamentosa dos $\mathrm{p}$ rasitos do paludismo humano configura um sério obstáculo clinico e um dos maiores entraves à erradicação e ao controle da endemia em várias regiões dos trópicos. A questão continua crescente nas áreas endêmicas, zonas rurais, onde a facilidade do $P$. falciparum superar os efeitos tóxicos das drogas destinadas a bloquear o seu desenvolvimento, alia-se à carência de médicos e à pobreza que induzem ao uso indiscriminado e arbitrário dos medicamentos.

A mefloquina, DL-erythro-2,8-bis (trifluorome thyl- $a$-(2-piperidyl)-4-quinolinemethanol hydrochloridrato, é um antimalárico sintetizado pelo Instituto Walter Reed que tem obtido sucesso no tratamento da doença em várias partes do mundo, inclusive onde o plasmódio é plurirresistente às drogas de uso corrente.

$O$ presente trabalho representa o primeiro teste terapêutico com a droga no Estado de Rondônia, situado na Amazônia Ocidental brasileira, considerada região malarigena hiperendêmica e, também, caracterizada como área de $P$. falciparum cloroquino-resistente, cujos resultados preliminares já haviam sido apresentados anteriormente ${ }^{14}$.

\section{MATERIAL E MÉTODOS}

$O$ tratamento com a mefloquina foi realizado em 1980 no Hospital Geral do 5. Batalhão de Engenharia

Núcleo de Medicina Tropical e Nutrição da Universidade de Brasilia

70910 - Brasilia-DF

Recebido para publicaçāo em $8 / 7 / 88$. de Construção, do Exército, localizado em Porto Velho (Rondònia).

Tratamos 90 pacientes do sexo masculino, com a idade de 13 a 55 anos. Todos tinham parasitemia inicial entre 4.000 e 80.000 trofozoitos de $P$. falciparum por $\mathrm{mm}^{3} \mathrm{e}$, não haviam recebido nenhum antimalárico nos 30 dias anteriores à consulta. Não foram tratados pacientes com malária grave, com parasitemia acima de 80.000 parasitos por $\mathrm{mm}^{3}$ de sangue, febre a mais de 10 dias, temperatura axilar acima de $40^{\circ} \mathrm{C}$, presença de distúrbios neurológicos, icterícia, hemoglobinúria, desidratação severa, choque cardiovascular, sinais de diástase hemorrágica, vòmitos incoercíveis, viciados em drogas, alcoólatras ou com outras doenças associadas.

Os pacientes foram hospitalizados durante oito dias, obedecendo a seguinte rotina: no tratamento, realizava-se a anamnese e o exame físico compreendendo avaliação do estado geral, pulso radial $\mathrm{e}$ freqüencia respiratoria; fazia-se a palpação do figado, do baço e dos linfonodos e a ausculta dos pulmões e do coração. A seguir, colhia-se sangue por punção digital, para a gota espessa, corada pelo Giemsa, para contagem de parasitos por $\mathrm{mm}^{3}$ de sangue.

No dia seguinte, pela manhã, obtinha-se sangue para os exames hematológicos (velocidade de hemossedimentação, hemoglobina, hematócrito, leucograma, contagem de plaquetas e de reticulócitos) e bioquimicos (bilirrubina total, desidrogenase lática, fosfatase alcalina, transaminase oxalacética, transaminase pirúvica, creatinina, ureia e glicose) e amostra de urina para o exame sumario (pesquisa de proteinas, glicose, urobilinogènio, hemoglobina e análise do sedimento). 
Após a colheita dos materiais para os exames, os doentes ingeriam dois comprimidos e, no dia seguinte, outros dois.

A administração da mefloquina foi feita segundo o método randômico duplo cego, usando-se doses totais de $750 \mathrm{mg}, 1000 \mathrm{mg}$ e $1250 \mathrm{mg}$, em três grupos de pacientes. No primeiro dia, todos os pacientes receberam $750 \mathrm{mg}$ da droga. No segundo dia, o primeiro grupo recebeu placebo, o segundo grupo 250 $\mathrm{mg}$ e, o terceiro, $500 \mathrm{mg}$ de mefloquina.

O primeiro controle foi feito oito horas apos a tomada da primeira dose, compreendendo avaliação da parasitemia, interrogatório sobre queixas e exame fisico, repetidos diariamente no periodo da internação e nos controles posteriores realizados no $14 \circ^{\circ}, 21 \circ^{\circ}$ e 28 . dia após o tratamento. Durante a hospitalização, o exame de urina foi feito, diariamente e os exames hematológicos e bioquímicos no $4 \%^{\circ}$ e no $7 \circ$ dias.

As reações colaterais observadas foram anotadas em relação ao tipo, intensidade, época de aparecimento e duração.

Quando necessário, associavam-se outros antimaláricos ao tratamento.

\section{RESULTADOS}

Dos 90 pacientes iniciais, excluimos quatro porque vomitaram logo após a ingestão da 1 a dose do medicamento e 11 porque na revisão das làminas foi questionada a presença de $P$. vivax ao invés de $P$. falciparum. Assim, consideramos apenas os resultados obtidos em 75 pacientes.

Quanto à idade dos doentes o maior percentual $(38,7 \%)$ estaria na faixà etária de 20 a 29 anos (Tabela $1)$.

O seguimento após o tratamento compreendeu sete dias para 26 pacientes $(34,7 \%), 14$ dias para 12 doentes $(16,0 \%), 21$ dias para $14(18,7 \%)$ e, 28 dias para $23(30,7 \%)$.

No ato da admissão, o número de empaludados com temperatura axilar até $37^{\circ} \mathrm{C}$ e considerados sem febre, foi igual a $11(14,7 \%)$. Os demais apresentaramse com temperatura acima de $37^{\circ} \mathrm{C}$. Em somente 7 $(9,3 \%)$ dos casos, a febre persistiu após o terceiro dia e em nenhum após o quarto dia de tratamento (Tabela 2).

No momento da consulta, as queixas gastrintestinais iniciais registradas foram: anorexia, nauseas, hipocondrialgia, empachamento pos-prandial, diarrèia, epigastralgia e obstipaçào. Os sintomas neurológicos referidos, ao chegarem ao hospital, constaram de: cefaleia, tonturas, parestesias, zumbidos, escotomas, insònia, mialgias e visão turva. Evidentemente toda esta sintomatologia pré-tratamento foi atribuida ao quadro clinico da malária. Em $48,0 \%$ dos pacientes, as queixas desapareceram até o quarto dia de trata- mento (Tabela 3), acompanhando a regressão da febre. Um paciente que usou $1250 \mathrm{mg}$ de mefloquina continuou com tonturas durante todo o periodo de internamento; constatou-se ser ele um portador de diabete mellitus. Os sintomas que não desapareceram após o acompanhamento hospitalar relacionavam-se ao aparelho digestivo.

As alterações mais encontradas no exame sumário de urina foram traços de albumina, vestígios de hemoglobina e raros cilindros, as quais regrediram até o quarto dia.

O tratamento não modificou o quadro hematológico (velocidade de hemossedimentação, hemoglobina, hematócrito, número de hemácias, leucócitos e plaquetas) durante os sete primeiros dias de acompanhamento. Entretanto, com qualquer dose, houve um aumento sensivel do número de reticulócitos circulantes. No sétimo dia de controle, os reticulócitos estavam acima de 20,0\% em 57 casos (76,0\%).

$\mathrm{Na}$ bioquímica do sangue, não houve alterações nas dosagens de bilirrubinas, transaminases, ureia, creatinina e glicose. Contudo, no sétimo dia de controle, o tratamento aumentou ou manteve elevada a deidrogenase lática em 21 casos $(28,0 \%)$ e a fosfatase alcalina em $31(41,0 \%)$.

$\mathrm{Na}$ grande maioria dos casos $(68,0 \%)$, a parasitemia negativou-se nos très primeiros dias após inicio do tratamento (Tabela 4). Em mais $24,0 \%$, os trofozoitos de $P$. falciparum desapareceram no quarto dia e em $5,3 \%$ no quinto. No sexto dia ainda havia um paciente positivo e no sétimo outro. Nào houve diferença significante da negativaçào parasitèmica com as diferentes doses de mefloquina. Nenhum paciente apresentou trofozoito de $P$. falciparum durante o seguimento de 7 a 28 dias.

As reações colaterais à droga foram entre discretas e moderadas e infreqüentes, representadas por náuseas, obstipação, mialgia e astenia (Tabela 5). Em $72,0 \%$ dos tratados não se constatou nenhum efeito indesejável à droga.

\section{COMENTÁRIOS}

Semelhante ao que foi demonstrado por outros autores $4571213 \mathrm{em}$ outras regiōes do mundo, a mefloquina mostrou-se também efetiva no tratamento de portadores de malária por $P$. falciparum no Estado de Rondônia. Na Amazônia brasileira, Lopez Antuñano e Wernsdorfer 8 já haviam demonstrado, "in vitro", a suscetibilidade à mefloquina do $P$. falciparum cloroquino-resistente procedente de Boa Vista, Roraima. Alecrim ${ }^{1}$ tratou 22 pacientes de Rondònia, obtendo apenas très casos de resistència a nivel de $\mathbf{R}_{1}$ com a dose de $750 \mathrm{mg}$.

A rápida negativação da parasitemia efetivou-se até o 4 \% dia de tratamento, no máximo, coincidindo 
Santos JB, Prata A. Tratamento de 75 pacientes portadores de malária pelo P. falciparum emPorto Velho (RO) com mefloquina. Revista da Sociedade Brasileira de Medicina Tropical 21: 181-185, Out-Dez, 1988

Tabela 1 - Tratamento de 75 pacientes portadores de malária pelo P. falciparum, em Porto Velho(RO), com mefloquina. Distribuição segundo a faixa etária.

\begin{tabular}{lcc}
\hline $\begin{array}{c}\text { Faixa Etária } \\
\text { (anos) }\end{array}$ & Número & $\%$ \\
\hline $13-19$ & 16 & 21,3 \\
$20-29$ & 29 & 38,7 \\
$30-39$ & 16 & 21,3 \\
$40-49$ & 11 & 14,7 \\
$50-55$ & 03 & 4,0 \\
Total & 75 & 100,0 \\
\hline
\end{tabular}

Tabela 2 - Tratamento de 75 pacientes portadores de malária pelo P. falciparum, em Porto Velho (RO), com mefloquina. Desaparecimento da febre, com o tratamento.

\begin{tabular}{lccccc}
\hline Dia de desaparecimento & \multicolumn{5}{c}{ Dose de mefloquina } \\
\cline { 2 - 6 } da febre & $1250 \mathrm{mg}$ & $1000 \mathrm{mg}$ & $750 \mathrm{mg}$ & Total & $\%$ \\
\hline Sem febre & 5 & 2 & 4 & 11 & 14,7 \\
1. dia & 1 & 4 & 4 & 9 & 12,0 \\
2. dia & 12 & 10 & 9 & 31 & 41,3 \\
3. dia & 4 & 7 & 6 & 17 & 22,7 \\
4. dia & 2 & 1 & 2 & 5 & 6,7 \\
5. dia & 0 & 1 & 0 & 1 & 1,3 \\
6. dia & 0 & 0 & 1 & 1 & 1,3 \\
7. dia & 0 & 0 & 0 & 0 & 0,0 \\
\hline
\end{tabular}

Tabela 3 - Tratamento de 75 pacientes portadores de malária pelo P. falciparum, em Porto Velho(RO), com mefloquina. Desaparecimento dos sintomas com o tratamento.

\begin{tabular}{lccccr}
\hline Dia de desaparecimento & \multicolumn{5}{c}{ Dose de mefloquina } \\
\cline { 2 - 6 } dos sintomas & 1250mg & $1000 \mathrm{mg}$ & $750 \mathrm{mg}$ & Total & $\%$ \\
\hline 10 dia & 1 & 3 & 2 & 6 & 8,0 \\
2. dia & 3 & 7 & 4 & 14 & 18,7 \\
3. dia & 4 & 2 & 1 & 7 & 9,3 \\
4. dia & 1 & 3 & 5 & 9 & 12,0 \\
5. dia & 3 & 3 & 2 & 8 & 10,7 \\
6. dia & 5 & 4 & 4 & 13 & 17,3 \\
7. dia & 3 & 3 & 5 & 11 & 14,7 \\
Nào desapareceram & 3 & 0 & 3 & 6 & 8,0 \\
Sem sintomas & 1 & 0 & 0 & 1 & 1,3 \\
\hline
\end{tabular}

com o tẹmpo de elevação do pique de concentração da droga no sangue que se faz ate a 72 a hora ${ }^{5}$. O desaparecimento da febre e dos sintomas neurológicos acompanharam o declinio da parasitemia. A persistência de alguns sintomas gastrointestinais após a suspensão da droga provavelmente indica a existèn- cia de problemas digestivos crònicos, comuns na area, onde as hepatopatias e as parasitoses intestinais sảo muito freqüentes, os quais estariam associados à infecção palúdica.

Contrario a outros trabalhos 14515 não houve diferenças significantes nos resultados obtidos com as 
Santos JB, Prata A. Tratamento de 75 pacientes portadores de malária pelo P. falciparum emPorto Velho (RO) com mefloquina. Revista da Sociedade Brasileira de Medicina Tropical 21: 181-185, Out-Dez, 1988

Tabela 4 - Tratamento de 75 pacientes portadores de malaria pelo P. falciparum, em Porto Velho (RO), com mefloquina. Negativação da parasitemia, com o tratamento.

\begin{tabular}{lccccc}
\hline & \multicolumn{5}{c}{ Dose de mefloquina } \\
\cline { 2 - 6 } Dia da negativação & $1250 \mathrm{mg}$ & $1000 \mathrm{mg}$ & $750 \mathrm{mg}$ & Total & $\%$ \\
\hline 1. dia & 1 & 0 & 0 & 1 & 1,3 \\
2. dia & 6 & 4 & 7 & 17 & 22,7 \\
3. dia & 10 & 13 & 10 & 33 & 44,0 \\
4. dia & 6 & 6 & 6 & 18 & 24,0 \\
5: dia & 0 & 2 & 2 & 4 & 5,3 \\
6. dia & 1 & 0 & 0 & 1 & 1,3 \\
7. dia & 0 & 0 & 1 & 1 & 1,3 \\
\hline
\end{tabular}

Tabela 5 - Tratamento de 75 pacientes portadores de malária pelo $P$. falciparum, em Porto Velho(RO), com mefloquina. Reaçōes colaterais.

\begin{tabular}{lccccc}
\hline & \multicolumn{5}{c}{ Dose de mefloquina } \\
\cline { 2 - 6 } Reações Colaterais & $1250 \mathrm{mg}$ & $1000 \mathrm{mg}$ & $750 \mathrm{mg}$ & Total & $\%$ \\
\hline Tontura & 6 & 3 & 4 & 13 & 17,3 \\
Diarreia & 2 & 2 & 4 & 8 & 10,7 \\
Náusea & 0 & 3 & 0 & 3 & 4,0 \\
Obstipação & 0 & 1 & 0 & 1 & 1,3 \\
Mialgia & 0 & 1 & 0 & 1 & 1,3 \\
Astenia & 0 & 0 & 1 & 1 & 1,3 \\
Sem Reações & 17 & 18 & 19 & 54 & 72,0 \\
\hline
\end{tabular}

três doses empregadas. As reações colaterais têm sido infreqüentes, de pequena intensidade e controláveis 245715 .

Apesar da efetividade da mefloquina em dose única $^{34}$, Richle $^{15}$ mostrou, em camundongos infectados pelo $P$. berghei, que, em dose única, os efeitos da mefloquina são mais lentos do que os da cloroquina na mesma dose e, Desjardins e cols ${ }^{3}$ e Felix e Gentilini ${ }^{5}$ desaconselharam-na face às variações individuais da cinética e as condições alimentares em determinadas regiōes que poderiam interferir na absorção gastrintestinal da droga, gerando falhas no tratamento em dose única. Observamos que não houve nenhuma dificuldade de administração do medicamento em duas tomadas, incremento de efeitos secundários indesejáveis nem diminuiçào da eficácia curativa da mefloquina com esse esquema posológico.

Houve um acentuado estimulo à hematopoiese com o tratamento, refletido no aumento sensivel dos reticulócitos circulantes. Na Malásia, Ponnampa$\operatorname{lam}^{13}$ notou a subida dos niveis de hemoglobina com a terapèutica.
O aumento da desidrogenase lática e da fosfatase alcalina na vigència do tratamento, provavelmente, deva-se a conseqüència da própria hemólise da malária.

Pacientes acompanhados durante 28 dias não mostraram recrudescèncias nem sinais de reinfecção. A meia vida sangüinea da mefloquina foi avaliada em $13,89 \pm 5,31$ dias por Desjardins e Gentilini ${ }^{3}$ e, em mais de 20 dias por Felix e cols 5 .

A eficacia curativa da mefloquina em uma area de $P$. falciparum plurirresistente ${ }^{1}$, a facilidade de administração em doses praticamente isentas de efeitos colaterais e, a possibilidade de excelente profilático tornam esse medicamento de capital importància no controle da endemia na região. Entretanto, o seu emprego deverá ser vigiado e controlado pelas autoridaes sanitárias, evitando que o uso indiscriminado possa gerar a indesejável resistència já relatada na literatura 19101112 . Para diminuir essa possibilidade, concordamos com outros autores 61011 que a mefloquina deva ser usada somente para os casos cloroquino ou plurirresistentes, uma vez que as associa- 
Santos JB, Prata A. Tratamento de 75 pacientes portadores de malária pelo P. falciparum emPorto Velho (RO) com mefloquina. Revista da Sociedade Brasileira de Medicina Tropical 21: 181-185, Out-Dez, 1988

ções medicamentosas preconizadas 101112 como medida para reduzir o perigo da resistência tornarão o emprego da droga mais dispendioso e mais dificil nessa região da Amazônia.

\section{SUMMARY}

Mefloquine was evaluated for the treatment of falciparum malaria in 75 patients in Porto Velho, Rondonia. Each patient was randomly assigned to one of three treatment groups and treated under a double blind protocol with a total dose of $750,1000 \mathrm{or}$ $1250 \mathrm{mg}$ of mefloquine per person. There were no significant differences among the groups. By the thirdy day of treatment $76 \%$ of the patients were without fever and $68 \%$ were without parasites. The hemograms were normal except for an increase in the number of reticulocytes in $76 \%$ of the patients. The concentration of lactic dehydrogenase in serum was elevated in $28 \%$ of the patients and the concentration of alkaline phosphatase was elevated in $41 \%$. Mild side effects were encountered in $18 \%$ of the patients. None of the patients suffered a relapse of malaria. It is concluded that mefloquine is practical, efficacious and safe for the treatment of falciparum malaria.

Key words: Malaria. Mefloquine. Treatment.

\section{AGRADECIMENTOS}

Os autores agradecem aos Laboratórios Roche pelo fornecimento de mefloquina e auxílio para a realização do presente trabalho.

\section{REFERÊNCIAS BIBLIOGRÁFICAS}

1. Alecrim MGC. Estudo da Resistència do Plasmodium falciparum às drogas anti-maláricas in vivo e in vitro na Amazònia. Tese de mestrado. Universidade de Brasilia, 1981.

2. Clyde DF, McCarthy VC, Miller RM, Hornick RB. Supressive activity of mefloquine in sporozoite induced human malaria. Antimicrobial Agents and Chemotherapy 9: 384-386, 1976.

3. Desjardins RE, Pamplin CL, Von Bredow J, Barry $\mathrm{KG}$, Canfield $\mathrm{CJ}$. Kinetics of a new antimalarial, mefloquine. Clinical Pharmacology and Therapeutics 26: $372-$ 379,1979

4. Doberstyn EB, Phintuyothin P, Noeypatimanondh S, Teerakiartkamjorn C. Single-dose therapy of falciparum malaria with mefloquine or pyrimethamine-sulfadoxine. Bulletin of the World Health Organization 57: 275-279, 1979.

5. Felix H, Gentilini M. La mefloquine-Rapport Preliminaire. Deuxième Congrés de la Societé Mediterranéenne de Chimiotherapie, 1980.

6. Fernex M. Urgent need to develop new antimalarials. Schweizerische Rundscham für Medizin. 70: 1025-1032, 1981.

7. Hall AP, Doberstyn EB, Karnchanachetanee C, Samransamruajkit S, Laixuthai B, Pearlman EJ, Lampe RM, Miller CF, Phintuyonthin P. Sequential treatment with quinine and mefloquine or quinine and pyrimethaminesulfadoxine for falciparum malaria. British Medical Journal 1: 1626-1628, 1977.

8. López Antuñano FJ, Wernsdorfer WH. In vitro response of chloroquine-resistant Plasmodium falciparum to mefloquine. Bulletin oi tive World Health Organization 57: 663-665, 1979.

9. Merkli B, Richle RW. Studies on the resistance to single and combined antimalarials in the Plasmodium berghe $i$ mouse model. Acta Tropica 37: 228-231, 1980

10. Merkli B, Richle R, Peters W. The inhibitory effect of a drug combination on the development of mefloquine resistance in Plasmodium berghei. Annals of Tropical Medicine and Parasitology 74: 1-9, 1980.

11. Peters W, Howells RE, Portus J, Robinson BL, Thomas $\mathrm{S}$, Warhurst DC. The chemotherapy of rodent malaria, XXVII. Studies on mefloquine (WR 142, 490). Annals of Tropical Medicine and Parasitology 71: 407-417, 1977.

12. Peters E, Portus J, Robinson BL. The chemotherapy of Rodent malaria, XXVIII. The development of resistance to mefloquine (WR 142, 490). Annals of Tropical Medicine and Parasitology 71: 419-427, 1977.

13. Ponnampaham JT. Mefloquine in the treatment of malaria - a preliminary study. $10^{\text {th }}$ Congress of Tropical Medicine and Malaria. Manila/Philippines p. 9-15, 1980.

14. Prata A, Santos JB, Chagas FP, Reis de Oliveira M, Paiva MC, Ferreira I. Double blind study with different doses of mefloquine (RO-21-5998) for the treatment of falciparum malaria in Brazil. Annals of the $12^{\text {th }}$ International Congress of Chemotherapy Florence/Italy 2: 1045-1046, 1981.

15. Richle RW. Chemotherapeutic activity of mefloquine hydrochloride in experimental Plasmodium berghei malaria in mice. Current Chemotherapy and Infectious Disease, American Society of Microbiology p. 11051107, 1980 .

16. Trenholme GM, Williams RL, Desjardins RE, Frischer H. Larson PE, Rieckmann KH. Mefloquine (WR 142, 490 ) in the treatment of human malaria. Science 190 : 792-794, 1975. 